\title{
Pseudomonas aeruginosa Implant-Associated Bone and Joint Infections: Experience in a Regional Reference Center in France
}

\begin{abstract}
Matteo Cerioli ${ }^{1}$, Cécile Batailler ${ }^{1,2,3}$, Anne Conrad ${ }^{1,2,3,4}$, Sandrine Roux ${ }^{1,2,3}$, Thomas Perpoint ${ }^{1,3}$, Agathe Becker ${ }^{1,3}$, Claire Triffault-Fillit ${ }^{1,3}$, Sebastien Lustig ${ }^{1,2,3}$, Michel-Henri Fessy 1,2,3, Frederic Laurent ${ }^{1,2,3,4}$, Florent Valour ${ }^{1,2,3,4}$, Christian Chidiac ${ }^{1,2,3,4}$ and Tristan Ferry ${ }^{1,2,3,4 *}$ on behalf of the Lyon Bone Joint Infection Study Group

${ }^{1}$ Hospices Civils de Lyon, Lyon, France, ${ }^{2}$ Université Claude Bernard Lyon 1, Lyon, France, ${ }^{3}$ Centre Interrégional de Référence des Infections Ostéo-articulaires complexes (CR/OAc Lyon), Hospices Civils de Lyon, Lyon, France, ${ }^{4}$ Centre International de Recherche en Infectiologie, CIRI, Inserm U1111, CNRS UMR5308, ENS de Lyon, UCBL1, Lyon, France
\end{abstract}

\section{OPEN ACCESS}

Edited by:

Murat Akova,

Hacettepe University, Turkey

Reviewed by:

Guangqiang Wang,

University of Shanghai for Science and

Technology, China

Werner Zimmerli,

Cantonal Hospital Baselland

(KSBL), Switzerland

Gennaro De Pascale,

Catholic University of the Sacred

Heart, Italy

*Correspondence: Tristan Ferry

tristan.ferry@univ-lyon1.fr

Specialty section:

This article was submitted to Infectious Diseases - Surveillance,

Prevention and Treatment,

a section of the journal

Frontiers in Medicine

Received: 19 November 2019

Accepted: 22 September 2020

Published: 26 October 2020

Citation:

Cerioli M, Batailler C, Conrad A,

Roux S, Perpoint T, Becker A, Triffault-Fillit C, Lustig S, Fessy M-H,

Laurent $F$, Valour $F$, Chidiac $C$ and

Ferry T (2020) Pseudomonas aeruginosa Implant-Associated Bone and Joint Infections: Experience in a Regional Reference Center in France.

Front. Med. 7:513242.

doi: 10.3389/fmed.2020.513242
Background: $P$. aeruginosa implant-associated bone and joint infections (BJI) is considered to be one of the most difficult to treat BJl. The data focusing specifically on this pathogen are sparse, and it seems difficult to extrapolate the results obtained with Enterobacteriaceae.

Methods: We performed a retrospective observation study of all $P$. aeruginosa implant-associated BJl diagnosed at our institution from 2011 to 2018. We defined failure as any type of relapse, including persistence of the same $P$. aeruginosa, superinfection by another organism(s) or any other cause of relapse such as the need for a subsequent surgery. Nonparametric statistical methods were used to compare the study groups and Kaplan-Meier curves and multivariate Cox analysis and were used to detect determinants associated with treatment failure.

Results: A total of 90 patients (62\% men, median age 60 years IQR 47-72) including $30(33 \%)$ prosthetic-joint infections and 60 (66\%) other implant-associated BJls were studied. Most of them were acute (62\%). During the prolonged follow-up, (median 20 months; IQR 9-37), 23 patients (26\%) experienced treatment failure. Optimal surgical treatment (DAIR for acute forms, explantation, 1-stage or 2-stage exchange for others) was significantly associated with a higher success rate in the univariate analysis $(p=0.003)$. Sixty-four $(71 \%)$ patients received effective initial treatment against $P$. aeruginosa administered and 81 of them (90\%) did for at least 3 weeks: both these parameters correlated with a higher success rate. In the multivariate Cox-analysis optimal surgical treatment, IV effective treatment of at least 3 weeks and treatment with ciprofloxacin for at least 3 months proved to be independently associated to a better outcome in patients with $P$. aeruginosa implant-associated BJl.

Conclusion: $P$. aeruginosa implant-associated $\mathrm{BJ}$ is one of the most difficult-to-treat BJl, with a strong impact on the prognosis of the surgical strategy. An effective initial IV antibiotic treatment for at least 3 weeks seems to be required, followed by oral ciprofloxacin for a total duration of 3 months.

Keywords: pseudomonas, osteomyelitis, ciprofloxacin, implant-associated bone infections (IABI), bone and joint infection 


\section{INTRODUCTION}

Implant-associated bone and joint infection (BJI) is an uncommon, but dreadful complication of arthroplasties and orthopedic trauma. Despite technological and medical effort in preventing such conditions, the amount of implant-associated infections is growing because of the increasing number of implant devices $(1,2)$. According to Zimmerli et al., the infection rate during the first 2 years varies according to the site and it is $<1 \%$ in hip and shoulder prostheses, $<2 \%$ in knee prostheses, and $<9 \%$ in elbow prostheses (3). With regard to internal fixation devices, about $5-10 \%$ becomes infected, with a significant disproportion between the major rate of infection after internal fixation of grade 3 open fractures (which may exceed $30 \%$ ) against the $0.5-2 \%$ rate of infection after internal fixation of closed wounds (1).

The most frequently isolated microorganisms in implantassociated BJI are Gram-positive cocci, with Staphylococcus aureus being the most recurrent cause, while Gram-negative bacteria (GNB) are responsible for $10-23 \%$ of all episodes, causing most often acute and polymicrobial infections (1, 3-6). Even if GNB cause a minor- yet, substantial- proportion of all implant-associated BJI, they draw the attention of the medical community in light of the fact that the treatment is rather complicated and they show a less optimal outcome with longer hospitalizations -and higher costs- due to their peculiar virulence, their growing resistance to antibiotics and the comorbidities of the patients they usually infect, generally immunocompromised ones (6-9). P. aeruginosa is a particular GNB, commonly considered as non-fermenting bacterium, that causes 5 to $20 \%$ of the GNB infections, and recent data revealed that $14 \%$ of patients with open fracture suffered from $P$. aeruginosa infection $(10,11)$. $P$. aeruginosa is considered as one of the most difficult-to-treat $\mathrm{GNB}$, as a result of its growing rate of multidrug-resistant strains and its ability to develop particular virulence and persistence mechanisms, such as biofilm formation and production of small colony variants (12).

Treatment strategies for staphylococcal implant-associated BJI are somewhat standardized, with a clear percentage of success, since they represent a significant cause of infection, which makes them easier to sample and study $(1,13,14)$. On the contrary, our path to mastering Gram-negative implant-associated infections has been paved with scarce published experience, mostly retrospective studies, which showed inconsistent data concerning surgical and antimicrobial treatment $(6-8,15-18)$. Currently, guidelines for antibiotic treatment of GNB implant-associated infections recommend beta-lactams and ciprofloxacin $(1,13)$. This has also been supported by a large multicentre study which deals with acute GNB infections treated with debridement antibiotics and implant retention (DAIR), reporting a 79\% success rate in ciprofloxacinsusceptible GNB PJI (19). In this very same setting, P. aeruginosa caused up to $20 \%$ of the GNB infections (19). Of note, none of these studies focused specifically on $P$. aeruginosa infections.

To our knowledge, along the years there have been just a few publications with in vitro studies supporting the role of fluoroquinolones against $P$. aeruginosa (20-22). However, some antimicrobial combinations, such as cefepime-ciprofloxacin and ceftazidime-ciprofloxacin, have been reported as successful options in the treatment of $P$. aeruginosa bone and joint infections $(7,16)$. Moreover, ciprofloxacin has been connected to a better treatment outcome when administered in case of susceptible GNB $(15,17,23)$ and also of $P$. aeruginosa $(19,24)$.

The aims of the present study are to review our experience with the treatment of acute, delayed or chronic implantassociated $P$. aeruginosa $\mathrm{BJI}$, and to analyze the impact of optimal surgical treatment, effective antimicrobial IV therapy and ciprofloxacin use on the prognosis.

\section{MATERIALS AND METHODS}

\section{Study Design and Population}

We performed a retrospective study at the Croix Rousse hospital (Hospices Civils de Lyon, France), that is the national French reference center for osteoarticular infections of the South-East region (CRIOAc Lyon; http://www.crioac-lyon.fr). We included all patients, independently of time on follow-up, with $P$. aeruginosa implant-associated infection managed in our institution between January 2011 and June 2018. All cases present in this cohort were discussed and handled thanks to the cooperation of our multidisciplinary group. Data were obtained from the electronic and written medical records, collected into a Microsoft Access Database. This study is subject to declaration with the local Commission for Data Protection and Liberties under the $\mathrm{n}^{\circ} 18-176$ and is registered on ClinicalTrial under the $\mathrm{n}^{\circ} \mathrm{NCT} 03624855$.

\section{Definitions}

Implant-associated infection caused by $P$. aeruginosa was diagnosed according to the definition of organ/space surgical site infection proposed by the CDC (25) and also fulfilled the IDSA definition for patients with PJI and the new definition proposed by Metsemakers et al. for patients with internal fixation associated infections $(13,26)$. We identified as hematogenous acute BJIs those cases in which the patient had a normal joint function after the implantation, but experienced a sudden onset of symptoms more than 3 months after the index surgery, as previously reported by Wouthuyzen-Bakker et al. (27). At least one positive sample with $P$. aeruginosa in culture from deep perioperative samples was required.

Implant-associated infections in this study were defined as "early" if they occurred within 1 month from the date of implantation, "delayed" if they occurred between 1 and 3 months from the date of implantation and "chronic" if the onset of symptoms was $>3$ months from the date of implantation.

Treatment failure was defined as any type of relapse of implant-associated infection including persistence (new surgery with $P$. aeruginosa in culture), superinfection [isolation of another $\operatorname{organism}(\mathrm{s})]$ or any other cause of relapse such as the need for a subsequent surgery. Treatment was considered successful if the infection was in remission at the end of the course of antibiotics and during the entire usual follow-up in our institution. In case of need, suppressive therapy was undertaken 
for the treating physician to prolong the antibiotic treatment indefinitely in patients at high risk of persistence and relapse.

Optimal surgical treatment was evaluated according to the type of surgery and the timing of the infection. In case of acute infections, we defined "DAIR" as an optimal choice of intervention if performed within 1 month following the date of implantation and for patients with hematogenous infection. If the $P$. aeruginosa implant-associated BJI was itself a superinfection on an implant previously infected by another microorganism, and if the current episode of the infection was asymptomatic and discovered accidentally on systematic bone biopsies (i.e., without clinical signs of infection), we reckoned the surgical treatment as optimal independently from the timing. While if the superinfection was accompanied by the onset of new clinical symptoms or by the worsening of the patient conditions, we assessed as optimal only the surgical treatment which was undertaken within 1 month from the previous surgery.

Effective initial antibiotic treatment against $P$. aeruginosa was defined by the use of an active IV beta-lactam drug, based on drug-susceptibility on the antibiogram.

According to the classification of the Common Terminology Criteria for Adverse Events (CTCAE), serious adverse events (SAE) were defined as CTCAE grade 3-5 (28). All SAE were reviewed by a pharmacist and were attributed (or not) to the antibiotic on $P$. aeruginosa.

\section{Statistical Analysis}

Descriptive statistics were used to estimate the frequencies of the study variables, described as effective (\%) for dichotomous values and medians [interquartile range (IQR)] for continuous values. For the percentage calculation of each variable, the number of missing values was excluded from the denominator. Nonparametric statistical methods were used to compare the study groups (chi-square test, Fisher's exact test, or MannWhitney $\mathrm{U}$ test, as appropriate). Univariate Cox analysis and Kaplan-Meier curves (using the log-rank test) were used to determine determinants associated with treatment failure. Multivariate Cox analysis that includes significant determinants identified in the univariate analysis was performed, by adopting a ratio of 10 events per independent variable to avoid overfitting (maximum of three variables in the present study, selection based on the univariate analysis). A $p$-value of $<0.05$ was considered significant. Statistical analyses were performed using SPPS Statistics Base 17.0 (Softonic International, San Francisco, CA, USA).

\section{RESULTS}

Among the 1,638 implant-associated BJI occurring over the 7 -year study period, 90 patients (5.5\%) from the beginning of 2011 to end of 2017 were infected by Pseudomonas aeruginosa (including 30 with a PJI) according to our definition and were included. Basic demographic information can be found in Table 1.

Twenty-five patients experienced 28 adverse events during a course of treatment with antibiotics that were active on Pseudomonas aeruginosa (13 were SAE, 16 caused the
TABLE 1 | Characteristics of the 90 patients with $P$. aeruginosa implant-associated BJl according to the outcome.

\begin{tabular}{|c|c|c|c|c|}
\hline Characteristics & $\begin{array}{c}\text { Whole } \\
\text { population } \\
(n=90)\end{array}$ & $\begin{array}{l}\text { Failure } \\
(n=23)\end{array}$ & $\begin{array}{c}\text { Remission } \\
(n=67)\end{array}$ & $p^{a}$ \\
\hline Age in years (median, IQR) & $60(47-72)$ & $61(43-74)$ & $59(47-72)$ & 0.90 \\
\hline Male sex (n, \%) & $56(62)$ & $17(74)$ & $39(58)$ & 0.18 \\
\hline $\mathrm{BMI} \geq 30(n, \%)$ & $24(28)$ & $6(29)$ & $18(29)$ & 1 \\
\hline Active smoking ( $n, \%)$ & $29(35)$ & $10(44)$ & $19(32)$ & 0.34 \\
\hline Score ASA > 2 (n, \%) & $30(34)$ & $8(35)$ & $22(33)$ & 0.90 \\
\hline Score Charlson > 4 (n, \%) & $24(27)$ & $7(30)$ & $17(25)$ & 0.64 \\
\hline $\begin{array}{l}\text { Previous infection at the } \\
\text { same site }(n, \%)\end{array}$ & $19(21)$ & $6(26)$ & $13(19)$ & 0.50 \\
\hline Prosthesis (n, \%) & $30(33)$ & $7(30)$ & $23(34)$ & 0.73 \\
\hline $\begin{array}{l}\text { Age of implant in days } \\
\text { (median, IQR) }\end{array}$ & 47 (21.7-247.5) & $40(21-222)$ & $63(26-798)$ & 0.29 \\
\hline \multicolumn{5}{|l|}{ Type of infection $(n, \%)$} \\
\hline Acute & $56(62)$ & $14(61)$ & $42(63)$ & 0.98 \\
\hline Sub-acute & $8(9)$ & $2(9)$ & $6(9)$ & \\
\hline Chronic & $26(29)$ & $7(30)$ & $19(28)$ & \\
\hline Polymicrobial infection ( $n, \%)$ & $66(73)$ & $18(78)$ & $48(71)$ & 0.54 \\
\hline $\begin{array}{l}\text { BJl due to } P \text {. aeruginosa } \\
\text { ciprofloxacin-resistant }(n, \%)\end{array}$ & $11(12)$ & 9 (39) & 2 (3) & $<0.001$ \\
\hline $\begin{array}{l}\text { Optimal surgical treatment }{ }^{b} \\
(n, \%)\end{array}$ & $54(64)$ & $9(39)$ & $45(72)$ & 0.004 \\
\hline $\begin{array}{l}\text { Effective initial IV treatment }{ }^{\mathrm{C}} \\
(n, \%)\end{array}$ & $64(71)$ & $12(52)$ & $52(77)$ & 0.020 \\
\hline $\begin{array}{l}\text { Treatment with ciprofloxacin }{ }^{d} \\
(n, \%)\end{array}$ & 79 (88) & $13(57)$ & 66 (99) & $<0.001$ \\
\hline
\end{tabular}

$I Q R$, interquartile range.

a The $p$-value was determined by using chi-square or Fisher's exact test for categorical variables, Mann-Whitney $U$ test for continuous variables.

${ }^{b}$ After exclusion of the five patients who finally received suppressive antimicrobial therapy. c such as piperacilline, piperacilline-tazobactam, ceftazidime, cefepime, imipenemcilastatin, ceftolozane-tazobactam, ceftazidime-avibactam, based on the susceptibility on the antibiogram.

${ }^{d}$ After exclusion of the two patients that received ciprofloxacin as suppressive therapy.

interruption of the effective treatment, while six of them occurred in the ending phase of treatment and shortened merely the course of antibiotic hopefully without nicking the quality of the medical therapy).

Sites of infection were: knee (16), spine (15), hip (15), tibia (8), jaw (7), skull (6), ankle (5), femur (4), elbow (2), shoulder (2), foot (2), calcaneum (2), others (2), pubis (1), sacroiliac bone (1), humerus (1), patella (1), heel (1).

Fifty-eight (64\%) patients were considered to have optimal surgical treatment, including 21 DAIR for an acute infection, 2 had incomplete implant removal, 31 complete implant removal for a chronic infection, one complete ablation followed by amputation and one DAIR followed by amputation. Among the thirty-two (36\%) patients who do not meet these criteria, 20 had DAIR for a delayed or chronic infection, 1 had an incomplete implant removal, 13 had a complete implant removal. During a prolonged follow-up [median follow-up of 20 months (IQR, 9-37)]; 24 patients without failure were followed at least 2 years, 23 patients experienced a treatment failure: seven patients 
A

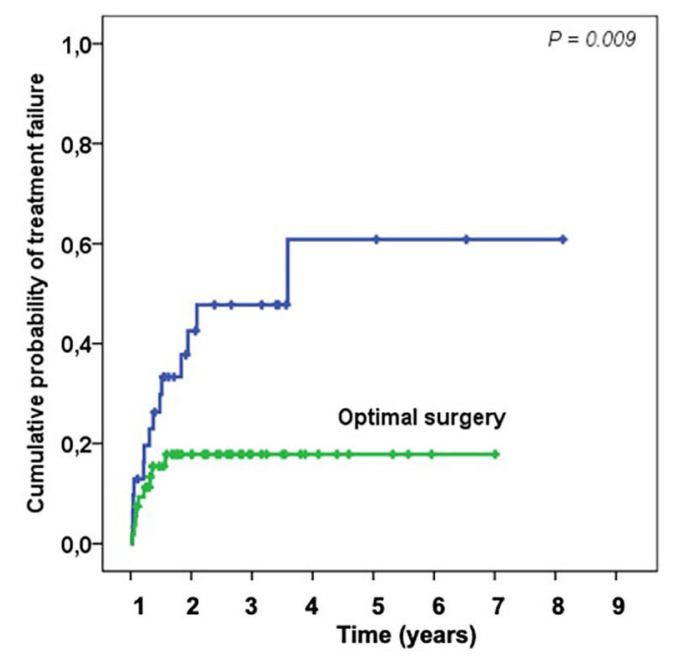

C

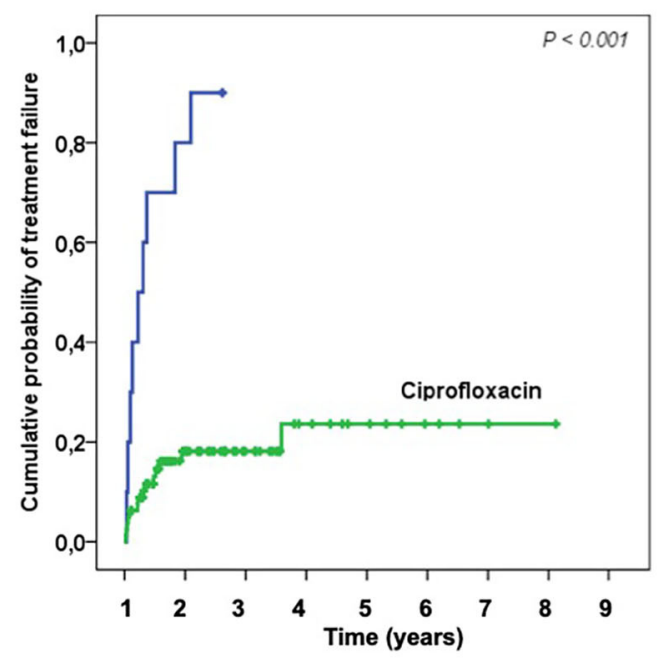

B

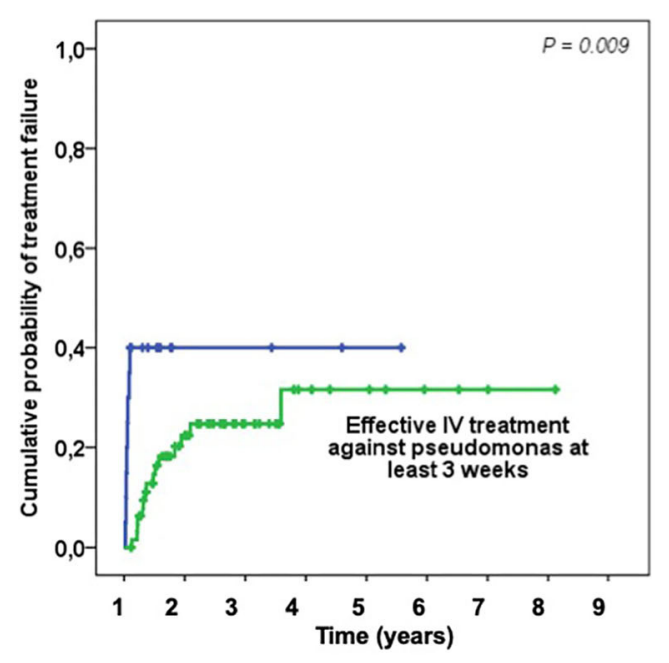

D

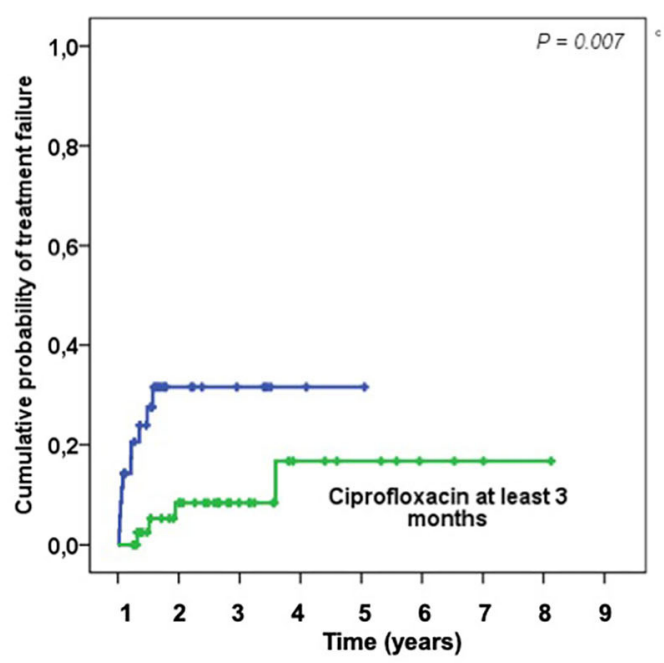

FIGURE 1 | Kaplan-Meier curves showing the probability of treatment failure depending on the surgical and medical treatments: Optimal surgery (A); Effective IV treatment against pseudomonas at least 3 weeks (B); Treatment with ciprofloxacin (C); Treatment with ciprofloxacin at least 3 months (D).

experience a persistence of $P$. aeruginosa after treatment, while 16 had a superinfection caused by another organism(s). Of note, 40 patients were lost to follow-up during the first 2 -years, but these patients were not excluded in the final analysis. Optimal surgical treatment was significantly associated with a higher success rate in the univariate analysis $(p=0.003)$ and in the Kaplan-Meyer survival curve (log-rank test, $\mathrm{p}=0.009$ ) (Table 1; Figure 1A). As long as it concerns the antimicrobial treatment, sixty-four (71\%) patients received effective initial treatment against $P$. aeruginosa administered by IV, while 26 (29\%) did not. Two patients with MDR P. aeruginosa (17\%) received ceftolozane/tazobactam or ceftazidime/avibactam. Not receiving an effective initial IV drug exposed the patient to an early failure (blue line in Figure 1B) and when we considered an IV treatment of at least 3 weeks, which was undertaken by 90 (81\%) patients, we found that it correlates with a higher success rate both in the univariate analysis $(p=0.020)$ and according to the Kaplan-Meyer curve (log-rank test, $p=0.009$ ) (Table 1 , Figure 1B). Eleven (12\%) patients had an infection due to a $P$. aeruginosa resistant to ciprofloxacin and this impacted as well $(p<0.001)$. In the end, we evaluated the effectiveness of the treatment with ciprofloxacin. Seventy-nine (88\%) patients received a course of therapy with ciprofloxacin and we found this as significantly associated with a higher success rate in the univariate analysis $(p<0.001)$ (Table 1; Figure 1C). Moreover, we observed a higher risk of failure if patients received $<3$ months of ciprofloxacin (log-rank test, $p=0.007$ ) (Figure 1D). In the multivariate Cox analyses, we included in the final model three variables which finally depict the optimal pattern of treatment: optimal surgical treatment, IV effective treatment of at least 3 weeks and treatment with ciprofloxacin for at least 3 months (Table 2 ). 
TABLE 2 | Multivariate Cox analysis that includes significant determinants for failure identified in the univariate analysis.

\begin{tabular}{|c|c|c|c|}
\hline & HR & $95 \% \mathrm{Cl}$ & $p$ \\
\hline Optimal surgical treatment* & 0.32 & $0.11-0.98$ & 0.045 \\
\hline IV effective treatment of at least 3 weeks* & 0.15 & $0.004-0.054$ & 0.003 \\
\hline Ciprofloxacin for at least 3 months* & 0.23 & $0.07-0.75$ & 0.015 \\
\hline
\end{tabular}

HR, Hazard ratio; 95\% Cl, 95\% confidence interval.

*After exclusion of the five patients who finally received suppressive antimicrobial therapy.

\section{DISCUSSION}

We have presented a case series of 90 implant-associated BJI caused by $P$. aeruginosa at our structure, managed at our institution during 2011-2017, which accounted for the 3\% of all $\mathrm{BJI}$ in this 7-year experience. To our knowledge, this is the largest and only study about implant-associated infections due to $P$. aeruginosa along with the one of Shah et al., which was only focused on PJIs (29).

Our data show that these infections are mostly acute and often polymicrobial, possibly due to the high comorbidity index of the patients involved and the opportunistic nature of a $P$. aeruginosa infection $(5,6,29)$. After a long-term follow-up, the remission rate of patients with a $P$. aeruginosa implant-associated BJI was $74 \%$ (67 out of 90), which is consistent with the results of Rodriguez-Pardo et al. on a smaller sample of $P$. aeruginosa cases $(n=43)$ included in a larger Gram-negative PJI study (19).

Surgical treatment is the cornerstone of Implant-associated infections and all of our patients underwent surgical procedures. Choosing the correct operation for the case among the number of options (DAIR, 1-stage or 2-stage exchange, palliative treatment) is much more subtle than what it looks like, and the decision should follow as possible the current guidelines. It must be a multidisciplinary, meticulous process, and it must take into account the patient status and integrate its functional prognosis in case of implant removal $(13,30)$. Lora-Tamayo et al. reported 33 patients with $P$. aeruginosa infected PJI and reached an overall success rate of $81 \%$ by treating early post-surgical and hematogenous infections with stable devices and good soft tissue conditions with DAIR, while they opted for an implant removal for the chronic cases (31). Ascione et al. described 11 cases of $P$. aeruginosa PJI, as part of a broader study, treated with DAIR ( $80 \%$ overall success rate) or 2-stage exchange for late infections (85\% overall success rate) (32). Once more, Veltman et al. presented a study on 12 early post-operative $P$. aeruginosa PJI treated with DAIR, reporting a success rate of $66 \%$ (24). These data are rather promising and in accordance with guidelines instructions, yet in contrast with the biggest $P$. aeruginosa PJI study (102 episodes in 91 patients), which pointed out a 5-year cumulative incidence of failure of $50 \%$ when treating PS PJI and an especially worse outcome for those treated with DAIR (2 year cumulative survival free rate of 26\%) (29). However, this study took into account infections occurred over a long period, therefore their optimal management was clearly limited by the lack of an established protocol, as proved by the fact that most patients who underwent DAIR had chronic infections (29). Among our patients the average duration of IV treatment was of 79 days [median 63 days, IQR (44-96)], while the average duration of the oral treatment with ciprofloxacin was of 111 days (median 79 days: IQR, 29-99). A recent study on 242 GNB PJIs, among which the $20 \%$ was caused by $P$. aeruginosa, DAIR was successful in $68 \%$ of cases, with an increase to $79 \%$ in ciprofloxacinsusceptible GNB PJI treated with ciprofloxacin (19). By judging the adequateness of all our patients' surgical treatment according to the current guidelines $(13,30)$, we found that optimal surgical treatment was significantly associated with a higher success rate, as previously reported in a study with $S$. aureus PJI (33).

Effective initial antibiotic betalactam treatment against $P$. aeruginosa proved to be a factor correlated with a better outcome $(p=0.020)$ in accordance to the guidelines and previous experiences $(7,13,16,34)$. Even if such antibiotics are recommended as initial therapy, their optimal duration is unclear. In patients with fluoroquinolone-susceptible Enterobacteriaceae, it is largely admitted that the duration of IV treatment could be shortened to 2 weeks $(20,23,35)$. In the study of Rodriguez-Pardo et al., P. aeruginosa cases were treated for a median of 60 days, with a combination of antibiotics in half of them, mainly an antipseudomonal beta-lactam plus ciprofloxacin. The median duration of the intravenous therapy (i.e., of the beta-lactam) was 18 days (19). As P. aeruginosa is considered to be a more difficult-to-treat bacterium in comparison with Enterobacteriaceae, as it has been speculated by some authors $(8,18)$, it is difficult to translate the results obtained with these latter bacteria exclusively, or with a minority of $P$. aeruginosa.

The treatment with ciprofloxacin was a factor significantly associated with a better outcome in our study. In the study of Shah et al., that included patients with Pseudomonas PJI in a period of time during which ciprofloxacin was not widely used during initial therapy (only nine out of the 102 received ciprofloxacin), the rate of success was particularly low (26\%) in patients treated with DAIR (29).

This finding is in line with what has already been suggested by Martinez-Pastor et al. (23), who examined GNB BJI and fluoroquinolones in general. As already proposed by RodriguezPardo et al., this finding supports the idea that the success of treatment depends on the susceptibility to this antibiotic and its use rather than on the causative microorganism (19). In this study, 28 of the $43 P$. aeruginosa cases received ciprofloxacin, for a median of 43 days. The overall success rate was $79 \%$ (33 of 42 cases), which increased to $88 \%$ (29 of 33) when only patients with ciprofloxacin were considered (19).

According to the literature, ciprofloxacin proved itself to be effective given its qualities (namely oral availability, diffusion into the bone, activity against biofilms) $(20,36)$. Concerning the optimal duration of the fluoroquinolone treatment in GNB BJI, it is probably ranged from 6 weeks -3 months, as we also found by checking the median duration of treatment in other studies 
$(19,24)$. In patients with fully susceptible Enterobacteriaceae native BJI, 6 weeks of treatment seem to be adequate. In patients with implant-associated BJI, a treatment course of 3 months has to be discussed, especially if $P$. aeruginosa is involved, as we found that such a duration was associated with a better outcome.

Of note, infection with a ciprofloxacin-resistant $P$. aeruginosa has a huge impact on the outcome: it has already been spotted as a risk factor advocating for implant removal even in acute infections (23), among the 11 patients infected with a ciprofloxacin-resistant $P$. aeruginosa in our study, nine experienced a failure. For this reason, fluoroquinolones should be avoided as empirical and initial therapy, yet they must be given only once having reduced the bacterial load, after a course of intravenous beta-lactam (15). There is no standard treatment for MDR GNB infection and $P$. aeruginosa is peculiarly challenging to treat, with scarce therapeutic options, that generally recur to combination of a new generation beta-lactam such as ceftolozane/tazobactam (37) or ceftazidime-avibactam (38) with colistin, which are inherently associated with high risk of toxic effects, while some in vitro and animal studies suggest a potential activity of the rifampin-colistin combination $(39,40)$.

Our work is an observational retrospective study that presents all the limitations implied by the inherent nature of this kind of study design. However, in the face of implant-associated infections, surgical and clinical management cannot be randomized; thus, observation studies are the best quality information we will ever have in this scenario. Secondly, it is crucial to focus accurately on patients with $P$. aeruginosa implant-associated BJI, as conclusions obtained with Enterobacteriaceae are not completely transposable. Finally, as $P$. aeruginosa implant-associated BJI is a potentially severe disease and as our center is a reference center for the management of BJI, we particularly try our best to follow these patient population. Even if the rate of lost to follow-up after 2 years was not negligible, very few data are lacking in our medical records, leading to interpretable results obtained from this study.

\section{CONCLUSIONS}

P. aeruginosa implant-associated BJI is one of the most difficultto-treat BJIs, with a strong impact on the prognosis of the surgical strategy. An effective initial IV antibiotic treatment for at least 3 weeks seems to be required, followed by oral ciprofloxacin for a total duration of 3 months.

\section{REFERENCES}

1. Trampuz A, Zimmerli W. Diagnosis and treatment of implant-associated septic arthritis and osteomyelitis. Curr Infect Dis Rep. (2008) 10:394403. doi: 10.1007/s11908-008-0064-1

2. del Pozo JL, Patel R. The challenge of treating biofilm-associated bacterial infections. Clin Pharmacol Ther. (2007) 82:204-9. doi: 10.1038/sj.clpt.6100247

3. Zimmerli W, Trampuz A, Ochsner PE. Prosthetic-joint infections. $N$ Engl J Med. (2004) 351:1645-54. doi: 10.1056/NEJMra0 40181

4. Murillo O, Grau I, Lora-Tamayo J, Gomez-Junyent J, Ribera A, Tubau F, et al. The changing epidemiology of bacteraemic osteoarticular infections

\section{DATA AVAILABILITY STATEMENT}

The datasets generated for this study are available on request to the corresponding author.

\section{ETHICS STATEMENT}

The studies involving human participants were reviewed and approved by Hospices Civils de Lyon Ethic Committee. Written informed consent from the participants' legal guardian/next of kin was not required to participate in this study in accordance with the national legislation and the institutional requirements.

\section{AUTHOR CONTRIBUTIONS}

MC wrote the first version of the manuscript. $\mathrm{MC}$ and TF performed the literature review. $\mathrm{CB}, \mathrm{AC}, \mathrm{SR}, \mathrm{TP}, \mathrm{AB}, \mathrm{CT}-\mathrm{F}$, SL, M-HF, FL, FV, CC, and TF participated to the patient care. All authors contributed to the article and approved the submitted version.

\section{ACKNOWLEDGMENTS}

Lyon Bone and Joint Infection Study Group: TF, Coordinator; TF, FV, TP, Patrick Miailhes, Florence Ader, SR, AB, CT-F, AC, Cécile Pouderoux, Marie-Elodie Langlois, Marielle Perry, Fatiha Daoud, Johanna Lippman, Evelyne Braun, CC, Infectious Diseases Specialists; SL, Elvire Servien, CB, Romain Gaillard, Stanislas Gunst, Julien Roger, Charles Fiquet, MH-F, Anthony Viste, Philippe Chaudier, Jean Luc Besse, Lucie Louboutin, Gaël Gaudin, Tanguy Ledru, Adrien Van Haecke, Quentin Ode, Marcelle Mercier, Florie AlechTournier, Sébastien Martres, Franck Trouillet, Cédric Barrey, Emmanuel Jouanneau, Timothée Jacquesson, Ali Mojallal, Fabien Boucher, Hristo Shipkov, Joseph, Chateau, Sophie Brosset, Philippe Céruse, Carine Fuchsmann, Arnaud Gleizal, Surgeons; Frédéric Aubrun, Mikhail Dziadzko, Caroline Macabéo, Anesthesiologists; FL, Laetitia Beraud, Céline Dupieux, Camille Kolenda, Jérôme Josse, Microbiologists; Fabien Craighero, Loic Boussel, Jean-Baptiste Pialat, Imaging; Isabelle Morelec, Marc Janier, Francesco Giammarile, Nuclear Medicine; Michel Tod, Marie-Claude Gagnieu, Sylvain Goutelle, PK/PD specialists; Eugénie Mabrut, Clinical research assistant and database manager.

in the early 21st century. Clin Microbiol Infect. (2015) 21:254.e1-8. doi: 10.1016/j.cmi.2014.09.007

5. Moran E, Masters S, Berendt AR, McLardy-Smith P, Byren I, Atkins BL. Guiding empirical antibiotic therapy in orthopaedics: the microbiology of prosthetic joint infection managed by debridement, irrigation and prosthesis retention. J Infect. (2007) 55:1-7. doi: 10.1016/j.jinf.2007. 01.007

6. Hsieh PH, Lee MS, Hsu KY, Chang YH, Shih HN, Ueng SW. Gram-negative prosthetic joint infections: risk factors and outcome of treatment. Clin Infect Dis. (2009) 49:1036-43. doi: 10.1086/605593

7. Legout L, Senneville E, Stern R, Yazdanpanah Y, Savage C, Roussel-Delvalez $\mathrm{M}$, et al. Treatment of bone and joint infections caused by Gram-negative 
bacilli with a cefepime-fluoroquinolone combination. Clin Microbiol Infect. (2006) 12:1030-3. doi: 10.1111/j.1469-0691.2006.01523.x

8. Zmistowski B, Fedorka CJ, Sheehan E, Deirmengian G, Austin MS, Parvizi J. Prosthetic joint infection caused by gram-negative organisms. J Arthroplasty. (2011) 26(6 Suppl.):104-8. doi: 10.1016/j.arth.2011.03.044

9. Sculco TP. The economic impact of infected joint arthroplasty. Orthopedics. (1995) 18:871-3.

10. Sudduth JD, Moss JA, Spitler CA, Pham VH, Jones LC, Brown JT, et al. Open fractures: are we still treating the same types of infections? Surg Infect. (2020). doi: 10.1089/sur.2019.140. [Epub ahead of print].

11. Triffault-Fillit C, Ferry T, Laurent F, Pradat P, Dupieux C, Conrad A, et al. Microbiologic epidemiology depending on time to occurrence of prosthetic joint infection: a prospective cohort study. Clin Microbiol Infect. (2018) 25:353-8. doi: 10.1016/j.cmi.2018.04.035

12. Pestrak MJ, Chaney SB, Eggleston HC, Dellos-Nolan S, Dixit S, Mathew-Steiner SS, et al. Pseudomonas aeruginosa rugose smallcolony variants evade host clearance, are hyper-inflammatory, and persist in multiple host environments. PLoS Pathog. (2018) 14:e1006842. doi: 10.1371/journal.ppat.1006842

13. Osmon DR, Berbari EF, Berendt AR, Lew D, Zimmerli W, Steckelberg JM, et al. Diagnosis and management of prosthetic joint infection: clinical practice guidelines by the Infectious Diseases Society of America. Clin Infect Dis. (2013) 56:e1-25. doi: 10.1093/cid/cis803

14. Zimmerli W, Widmer AF, Blatter M, Frei R, Ochsner PE. Role of rifampin for treatment of orthopedic implant-related staphylococcal infections: a randomized controlled trial. Foreign-Body Infection (FBI) Study Group. JAMA. (1998) 279:1537-41. doi: 10.1001/jama.279.19.1537

15. Aboltins CA, Dowsey MM, Buising KL, Peel TN, Daffy JR, Choong PF, et al. Gram-negative prosthetic joint infection treated with debridement, prosthesis retention and antibiotic regimens including a fluoroquinolone. Clin Microbiol Infect. (2011) 17:862-7. doi: 10.1111/j.1469-0691.2010.03361.x

16. Brouqui P, Rousseau MC, Stein A, Drancourt M, Raoult D. Treatment of Pseudomonas aeruginosa-infected orthopedic prostheses with ceftazidimeciprofloxacin antibiotic combination. Antimicrob Agents Chemother. (1995) 39:2423-5. doi: 10.1128/AAC.39.11.2423

17. Jaén N, Martínez-Pastor JC, Muñoz-Mahamud E, García-Ramiro S, Bosch J, Mensa, et al. Long-term outcome of acute prosthetic joint infections due to gram-negative bacilli treated with retention of prosthesis. Rev Esp Quimioter. (2012) 25:194-8.

18. Uçkay I, Bernard L. Gram-negative vs. gram-positive prosthetic joint infections. Clin Infect Dis. (2010) 50:795. doi: 10.1086/650540

19. Rodríguez-Pardo D, Pigrau C, Lora-Tamayo J, Soriano A, del Toro MD, Cobo J, et al. Gram-negative prosthetic joint infection: outcome of a debridement, antibiotics and implant retention approach. A large multicentre study. Clin Microbiol Infect. (2014) 20:O911-9. doi: 10.1111/1469-0691.12649

20. Tanaka G, Shigeta M, Komatsuzawa H, Sugai M, Suginaka H, Usui T. Effect of the growth rate of Pseudomonas aeruginosa biofilms on the susceptibility to antimicrobial agents: beta-lactams and fluoroquinolones. Chemotherapy. (1999) 45:28-36. doi: 10.1159/000007162

21. Reid G, Sharma S, Advikolanu K, Tieszer C, Martin RA, Bruce AW. Effects of ciprofloxacin, norfloxacin, and ofloxacin on in vitro adhesion and survival of Pseudomonas aeruginosa AK1 on urinary catheters. Antimicrob Agents Chemother. (1994) 38:1490-5. doi: 10.1128/AAC.38.7.1490

22. Yassien M, Khardori N, Ahmedy A, Toama M. Modulation of biofilms of Pseudomonas aeruginosa by quinolones. Antimicrob Agents Chemother. (1995) 39:2262-8. doi: 10.1128/AAC.39.10.2262

23. Martínez-Pastor JC, Muñoz-Mahamud E, Vilchez F, García-Ramiro S, Bori G, Sierra J, et al. Outcome of acute prosthetic joint infections due to gramnegative bacilli treated with open debridement and retention of the prosthesis. Antimicrob Agents Chemother. (2009) 53:4772-7. doi: 10.1128/AAC.00188-09

24. Veltman ES, Vos FJ, Meis JF, Goosen JH. Debridement, antibiotics and implant retention in early postoperative infection with Pseudomonas aeruginosa. J Infect. (2015) 70:307-9. doi: 10.1016/j.jinf.2014.10.002

25. Horan TC, Gaynes RP, Martone WJ, Jarvis WR, Emori TG. CDC definitions of nosocomial surgical site infections, 1992: a modification of CDC definitions of surgical wound infections. Am J Infect Control. (1992) 20:271-4. doi: 10.1016/S0196-6553(05)80201-9

26. Metsemakers WJ, Kortram K, Morgenstern M, Moriarty TF, Meex I, Kuehl $\mathrm{R}$, et al. Definition of infection after fracture fixation: a systematic review of randomized controlled trials to evaluate current practice. Injury. (2018) 49:497-504. doi: 10.1016/j.injury.2017.02.010

27. Wouthuyzen-Bakker M, Sebillotte M, Lomas J, Taylor A, Palomares EB, Murillo O, et al. Clinical outcome and risk factors for failure in late acute prosthetic joint infections treated with debridement and implant retention. J Infect. (2018) 78:40-7. doi: 10.1016/j.jinf.2018.07.014

28. Valour F, Karsenty J, Bouaziz A, Ader F, Tod M, Lustig S, et al. Antimicrobialrelated severe adverse events during treatment of bone and joint infection due to methicillin-susceptible Staphylococcus aureus. Antimicrob Agents Chemother. (2014) 58:746-55. doi: 10.1128/AAC.02032-13

29. Shah NB, Osmon DR, Steckelberg JM, Sierra RJ, Walker RC, Tande AJ, et al. Pseudomonas prosthetic joint infections: a review of 102 episodes. $J$ Bone Jt Infect. (2016) 1:25-30. doi: 10.7150/jbji.15722

30. Zimmerli W. Clinical presentation and treatment of orthopaedic implantassociated infection. J Intern Med. (2014) 276:111-9. doi: 10.1111/joim.12233

31. Lora-Tamayo J, Euba G, Ribera A, Murillo O, Pedrero S, GarcíaSomoza D, et al. Infected hip hemiarthroplasties and total hip arthroplasties: differential findings and prognosis. J Infect. (2013) 67:536-44. doi: 10.1016/j.jinf.2013.07.030

32. Ascione T, Pagliano P, Mariconda M, Rotondo R, Balato G, Toro A, et al. Factors related to outcome of early and delayed prosthetic joint infections. J Infect. (2015) 70:30-6. doi: 10.1016/j.jinf.2014.07.008

33. Bouaziz A, Uçkay I, Lustig S, Boibieux A, Lew D, Hoffmeyer P, et al. Non-compliance with IDSA guidelines for patients presenting with methicillin-susceptible Staphylococcus aureus prosthetic joint infection is a risk factor for treatment failure. Med Mal Infect. (2018) 48:207-11. doi: 10.1016/j.medmal.2017.09.016

34. Sendi P, Zimmerli W. Antimicrobial treatment concepts for orthopaedic device-related infection. Clin Microbiol Infect. (2012) 18:1176-84. doi: 10.1111/1469-0691.12003

35. Zimmerli W, Moser C. Pathogenesis and treatment concepts of orthopaedic biofilm infections. FEMS Immunol Med Microbiol. (2012) 65:158-68. doi: 10.1111/j.1574-695X.2012.00938.x

36. Rimmelé T, Boselli E, Breilh D, Djabarouti S, Bel JC, Guyo,t R., et al. Diffusion of levofloxacin into bone and synovial tissues. J Antimicrob Chemother. (2004) 53:533-5. doi: 10.1093/jac/dkh110

37. Hassan S, Kahn MD, Saraiya N, Nori P. Treatment of a complex orthopaedic infection due to extensively drug-resistant. BMJ Case Rep. (2018) 2018:bcr2017223202. doi: 10.1136/bcr-2017-223202

38. Schimmenti A, Brunetti E, Seminari E, Mariani B, Cambieri P, Orsolini P. Prosthetic joint infection from carbapenemase-resistant. Case Rep Infect Dis. (2018) 2018:1854805. doi: 10.1155/2018/1854805

39. Drapeau CM, Grilli E, Petrosillo N. Rifampicin combined regimens for gramnegative infections: data from the literature. Int J Antimicrob Agents. (2010) 35:39-44. doi: 10.1016/j.ijantimicag.2009.08.011

40. Forrest GN, Tamura K. Rifampin combination therapy for nonmycobacterial infections. Clin Microbiol Rev. (2010) 23:14-34. doi: 10.1128/CMR. 00034-09

Conflict of Interest: The authors declare that the research was conducted in the absence of any commercial or financial relationships that could be construed as a potential conflict of interest.

Copyright (c) 2020 Cerioli, Batailler, Conrad, Roux, Perpoint, Becker, TriffaultFillit, Lustig, Fessy, Laurent, Valour, Chidiac, Ferry. This is an open-access article distributed under the terms of the Creative Commons Attribution License (CC BY). The use, distribution or reproduction in other forums is permitted, provided the original author(s) and the copyright owner(s) are credited and that the original publication in this journal is cited, in accordance with accepted academic practice. No use, distribution or reproduction is permitted which does not comply with these terms. 